\title{
Redefining the Limits of Biochemistry in Multidrug Resistant Nematodes: Implications for Future Drug Development
}

\section{Hany Elsheikha and Cyril Rauch*}

School of Veterinary Medicine and Science, University of Nottingham, Sutton Bonington Campus, Loughborough, LE12 5RD, UK

\section{MDR and Nematode Parasites}

Chemotherapy remains the foremost available tool for the control of nematode parasites. However, the substantial use of anthelmintics, combined with a scarcity of new anthelmintic classes, has led to a gradual increase in the resistance of nematode parasites to these compounds. Multi-Drug Resistance (MDR) in economically important livestock nematodes, such as Haemonchus contortus enables the parasite to effectively 'escape' the effects of existing anthelmintics and represents a major obstacle to sustainable livestock farming and food production [1]. The growth in anthelmintic resistance has led to an increasing need for novel chemotherapeutic compounds.

Despite this unmet need, there has been a decline in anthelmintic research and development due to contraction of the industry, economic factors and regulatory requirements, among others [2]. Recently, two new anthelmintics have been released; however, resistant parasites are anticipated to appear in about a decade after the lunching of products, regardless their chemical structure or their mode of action. Hence, understanding the mechanisms of Drug Resistance (DR) in parasites is crucial for medical, veterinary, economic and eco-toxicological reasons and for maintaining the effectiveness of current anthelmintic drugs.

Among the different tactics by which nematode parasites tolerate the action of anthelmintic, those affecting influx and efflux are of special significance, as they limit the interaction of the chemical compound with its intracellular targets and hence, its lethal effects on the parasite. Mechanisms of drug evasion based on the intracellular extrusion of the drug and/or modification of target molecules have been described [3,4]. Cellular mechanisms related to metabolic activity have also been seen in eukaryotic systems, e.g. cancer cells. Recent observations suggest that such mechanism may occur in nematodes [5].

\section{Drug Transporter Mediating MDR: Beyond the Obvious}

Albeit an important body of works, fundamental questions remain unsolved that may, once understood allow us to design new therapeutic strategies. A structural study has enabled the molecular basis of P-gp (the archetypal drug transporter in cancer cells) to be defined with remarkable precision [6]. This study confirms that when ATP molecules bind to Pgp, conformational changes promote a "power stroke", leading to the passage of drugs from the membrane inner leaflet into the outer milieu [6,7]. Although the molecular model of Pgp has permitted a relatively simple representation of MDR in agreement with the usual concepts from the field of biochemistry, how a single protein can expel structurally different drugs, is still poorly understood. How P-gp recognizes hundreds of different hydrophobic drugs and pump them out of the cell is still debatable [8]. Nonetheless, there is something far more important at stake: the Pgp-like-mediated MDR model does not conform to the fundamental notion of specificity and seems to challenge the roots of biochemistry. This conceptual issue was noted very early in this field, and raised clearly by Paul Roepe [9]: “...MDR cells are resistant to, and/or exhibit decreased retention of literally hundreds of different hydrophobic compounds that are structurally divergent. Membrane transporters, like soluble enzymes, are exquisitely substrate-specific. If transporters were not specific, the cell would eventually become a high entropy chaotic mess, as there are no structural molecular motifs common to all the many different agents to which MDR cells are resistant. MDR protein is a very unusual enzyme with extraordinarily broad substrate recognition capabilities; that is, it violates the law of enzyme specificity".

Given the central importance of the notion of "specificity" in classical biochemistry, various models were put forward to explain MDR without absolutely requiring a drug-handling activity; by characterizing the interactions between the physico-chemical properties of drugs and biophysical changes recurrent in MDR cells, i.e. involving membrane potential [10], cytosolic $\mathrm{pH}[10,11]$ and membrane recycling $[12,13]$. Although conceptually satisfying and in accordance with the notion of specificity and hence, basic laws of biochemistry, it is worth noting that these models have their own limits and that they cannot rule out completely, the involvement of drug-handling by transporters. Are we back in a conceptual dead-lock?

Not necessarily, as recent works have demonstrated that membrane changes, reflected by alteration of parameters stated above (electric potential, $\mathrm{pH}$ and membrane recycling), may be central to maintain drugs in the membrane for a sufficient long time and hence allow drug pumping. Indeed, if we assume that, the specificity is not central to drug pumping, the only possibility for efficient drug extrusion relies on repeated collisions between a drug and the set of membrane transporters. For example, all chemists know that increasing the temperature accelerates the reaction rate. Albeit temperature is unlikely to be directly involved as such in drug pumping, it is worth mentioning that physicists have demonstrated a similar role to diffusion in two dimensions $[14,15]$. This would mean that extrusion is warranted by the diffusion properties and membrane changes. Looking at the MDR problem from this angle, opens new possibilities for the development of novel therapeutic strategies.

If the membrane diffusion is so important why not targeting the membrane itself to change the ability of transporters to interact with drugs. Doing so, we would impair the drug/transporter interaction allowing more drug uptake by cells. These membrane changes that seem so important in MDR have to be related to how parasites "handle"

*Corresponding authors: Cyril Rauch, School of Veterinary Medicine and Science, University of Nottingham, Sutton Bonington Campus, Loughborough, LE12 5RD, UK, Tel: 44-0-115 9516451; Fax: 44-0-115 9516440; E-mail: cyril.rauch@nottingham.ac.uk

Received October 17, 2012; Accepted October 17, 2012; Published October 19 2012

Citation: Elsheikha H, Rauch C (2012) Redefining the Limits of Biochemistry in Multidrug Resistant Nematodes: Implications for Future Drug Development. J Vet Sci Technol 3:e110 doi:10.4172/2157-7579.1000e110

Copyright: (c) 2012 Elsheikha H, et al. This is an open-access article distributed under the terms of the Creative Commons Attribution License, which permits unrestricted use, distribution, and reproduction in any medium, provided the original author and source are credited. 
Citation: Elsheikha H, Rauch C (2012) Redefining the Limits of Biochemistry in Multidrug Resistant Nematodes: Implications for Future Drug Development. J Vet Sci Technol 3:e110 doi:10.4172/2157-7579.1000e110

Page 2 of 2

their membrane, i.e. what happen to parasites' membrane when they reach the drug resistant state. Thus, if one wants to eradicate MDR, it is the membrane of parasites that needs to be focused upon and targeted by specific drugs.

\section{References}

1. Kaplan RM (2004) Drug resistance in nematodes of veterinary importance: a status report. Trends Parasitol 20: 477-481.

2. Woods DJ, Knauer CS (2010) Discovery of veterinary antiparasitic agents in the 21st century: a view from industry. Int J Parasitol 40: 1177-1181.

3. McCavera S, Walsh TK, Wolstenholme AJ (2007) Nematode ligand-gated chloride channels: an appraisal of their involvement in macrocyclic lactone resistance and prospects for developing molecular markers. Parasitology 134 $1111-1121$

4. Riou M, Guégnard F, Sizaret PY, Le Vern Y, Kerboeuf D (2010) Drug resistance is affected by colocalization of P-glycoproteins in raft-like structures unexpected in eggshells of the nematode Haemonchus contortus. Biochem Cell Biol 88: 459-467.

5. Kerboeuf D, Riou M, Neveu C, Issouf M (2010) Membrane drug transport in helminths. Anti-Infective Agents in Med Chem 9: 113-129.

6. Aller SG, Yu J, Ward A, Weng Y, Chittaboina S, et al. (2009) Structure of P-glycoprotein reveals a molecular basis for poly-specific drug binding. Science 323: 1718-1722.
7. Sauna ZE, Ambudkar SV (2007) About a switch: how P-glycoprotein (ABCB1) harnesses the energy of ATP binding and hydrolysis to do mechanical work. Mol Cancer Ther 6: 13-23.

8. Gottesman MM, Ambudkar SV, Xia D (2009) Structure of a multidrug transporter. Nat Biotechnol 27: 546-547.

9. Roepe PD (2000) What is the precise role of human MDR 1 protein in chemotherapeutic drug resistance? Curr Pharm Des 6: 241-260.

10. Wadkins RM, Roepe PD (1997) Biophysical aspects of P-glycoprotein-mediated multidrug resistance. Int Rev Cytol 171: 121-165.

11. Simon SM, Schindler M (1994) Cell biological mechanisms of multidrug resistance in tumors. Proc Natl Acad Sci U S A 91: 3497-3504.

12. Rauch C (2009) On the relationship between drug's size, cell membrane mechanical properties and high levels of multi drug resistance: a comparison to published data. Eur Biophys J 38: 537-546.

13. Rauch C (2009) Toward a mechanical control of drug delivery. On the relationship between Lipinski's 2 nd rule and cytosolic $\mathrm{pH}$ changes in doxorubicin resistance levels in cancer cells: a comparison to published data. Eur Biophys J 38: 829846.

14. Panagiotopoulou V, Richardson G, Jensen OE, Rauch C (2010) On a biophysical and mathematical model of Pgp-mediated multidrug resistance: understanding the "space-time" dimension of MDR. Eur Biophys J 39: 201-211.

15. Rauch C (2011) The "multi" of drug resistance explained by oscillating drug transporters, drug-membrane physical interactions and spatial dimensionality. Cell Biochem Biophys 61: 103-113. 